\title{
THE DIAGNOSIS OF EPIDEMIC MENINGITIS AND THE CONTROL OF ITS TREATMENT BY RAPID BAC- TERIOLOGIC AND SEROLOGIC METHODS
}

\author{
M. B. Coн en, (West Salem, Ohio) \\ AND \\ J. S. FLEMING, (Memphis, Tenn.) \\ First Lieutenants, M. R. C., U. S. Army
}

From the Laboratory of Evacuation Hospital No. 14 and the Bacteriologic Department of the Base Hospital, Camp Jackson, S. C.

There is no acute disease which requires more acumen for its diagnosis and treatment than epidemic meningitis. There are many abortive cases and many cases of undoubted meningococcus sepsis which can be diagnosed before the advent of meningitis. As success in treatment depends on early diagnosis, it is imperative that the laboratory be called on to aid at the earliest possible moment, as the final diagnosis is always made in the laboratory. To give proper aid the bacteriologist must have a well equipped laboratory and be familiar with rapid methods of culture of the meningococcus.

This organism has always been described as an aerobic gramnegative diplococcus and until recently only aerobic methods have been used for its isolation and cultivation. A number of special mediums have been advocated; their number attest to the difficulty in growing many strains. Attention has been called to the importance of the reaction of the medium and to the fact that the meningococcus will not usually grow when the reaction is over plus 0.5 acid to phenolphthalein.

All these painstaking methods are the result of the attempt to cultivate the meningococcus aerobically, as all have assumed it to be an obligate aerobe. This assumption is incorrect.

Many have studied aerobic and anaerobic organisms and the corresponding methods of cultures, but only a few have investigated the oxygen requirements of organisms which lie between the aerobic and anaerobic ones, and are usually known as facultative. There is a group of organisms which require for their optimum growth an atmosphere in which the percentage of oxygen is slightly less than that of air. The members of this group are usually spoken of as partial tension organisms, and the meningococcus belongs to this group. ${ }^{1}$

Received for publication Aug. 2, 1918.

1 Cohen, M. B., and Markle, L.: Jour. Am. Med. Assn., 1915, 67, p. 1302; Cohen, M. B.: Ibid., 1918, 70, p. 1999. 
When this basic fact is recognized, meningococcus culture becomes almost as simple as staphylococcus culture. One can isolate it on any enriched medium such as blood agar, the reaction of which may be as high as plus 1.0 acid to phenolphthalein.

Originally, partial tension cultures were made by attaching a slant containing the meningococcus material to a freshly inoculated agar slant of B. subtilis. It was assumed that the rapidly growing B. subtilis used up sufficient oxygen to make the gaseous medium of the proper oxygen tension for the meningococcus growth. That this is actually the case is shown by a series of experiments recently carried out: Several meningococcus strains were isolated from spinal fluids by the subtilis method. The cultures were emulsified in broth and a loopful of each was planted on each of several blood agar slants of the same batch of medium. One slant of each strain was incubated aerobically, one was connected with $\mathrm{B}$. subtilis, and the remainder were placed in jars which contained 10,20,30, 50 and $75 \% \mathrm{Co}_{2}$, the rest being in all cases air. All cultures were incubated for 24 hours at $37 \mathrm{C}$. and then examined. The following is a typical record:

June 20: Culture A isolated from spinal fluid by subtilis method. Standard loop of broth emulsion planted on 7 tubes of human blood agar with a reaction of +0.2 and incubated at $37 \mathrm{C}$.

June 21: Tube 1, aerobic: no growth.

Tube 2, subtilis : rich growth.

Tube 3, 10\% $\mathrm{CO}_{2}$ : very rich growth.

Tube $4,20 \% \mathrm{CO}_{2}$ : very rich growth.

Tube 5, 30\% $\mathrm{CO}_{2}$ : good growth.

Tube $6,50 \% \mathrm{CO}_{2}$ : scant growth.

Tube $7,75 \% \mathrm{CO}_{2}$ : several small colonies.

From a number of experiments it was determined that the optimum gas mixture varied from $10-30 \% \quad \mathrm{CO}_{2}$ with air, depending on the strain of the organism. As growth in $10 \% \mathrm{CO}_{2}$ was always as good as in any greater concentration, and always better than at normal oxygen pressure, a mixture of $10 \% \mathrm{CO}_{2}$ and $90 \%$ air was adopted as a routine for all meningococcus work.

These $\mathrm{CO}_{2}$ air mixtures were prepared as follows:

Jars of known capacity which could be tightly sealed were used to hold the cultures. The $\mathrm{CO}_{2}$ generated from weighed amounts of $\mathrm{Na}_{2} \mathrm{CO}_{3}$ by the action of sulphuric acid. By knowing the volume of the container and the per cent. of $\mathrm{CO}_{2}$ desired one determined the amount of $\mathrm{CO}_{2}$ needed. The exact amount of $\mathrm{Na}_{2} \mathrm{CO}_{3}$ necessary to produce this volume of gas was determined in the following manner: According to a well known law of physical chemistry (Gay Lussac) the molecular weight of any substance expressed in gm., occupies in the gaseous state at normal temperature and pressure 22.3 liters. As the molecular weight of $\mathrm{Na}_{2} \mathrm{CO}_{3}$ is $106,106 \mathrm{gm}$. will produce 22.3 liters of $\mathrm{CO}_{2}$. The amount necessary to produce $10 \%$ of any known volume 
is then easily figured. The variations in the amount of gas formed due to differences in temperature and pressure were ignored as the percentage of air from these factors is too small to be of any significance in an experiment of this kind.

Herrick $^{2}$ calls attention to the fact that epidemic meningitis begins as a meningococcus sepsis, and that for a period from a few hours to many days there is no definite localization in the meninges. It is imperative to make a diagnosis at this stage of the disease as by early massive serum treatment it may be possible to prevent the onset of meningitis or at least to render the disease less severe. During this stage the spinal fluid is usually clear and meningococci may or may not be found in direct smears or by the usual method of culture. In 1916 Cohen and Markle reported 15 positive cultures by the subtilis method from clear spinal fluid. Four positive cultures were obtained from the same series aerobically, but direct smears were constantly negative. Partial tension cultures should always be made from such clear fluids. It is of the utmost importance to make blood cultures also. From $10-20 \mathrm{c}$ c of blood are removed from a vein and are placed in 2 flasks of neutral dextrose-infusion broth. These flasks are incubated in an atmosphere of $10 \% \mathrm{CO}_{2}$. Baeslack ${ }^{3}$ and associates found meningococci in the blood in $36 \%$ of early cases. This percentage can probably be increased by the method described in the foregoing.

In the cases with well developed meningitis the meningococcus can be usually demonstrated in direct smears from the spinal fluid. To isolate it the spinal fluid is centrifuged and a loopful of the sediment is planted on each of 4 or 5 tubes of human blood agar or several loopfuls are spread on the surface of a blood-agar plate. These cultures are then placed in a jar, the proper amount of $\mathrm{Na}_{2} \mathrm{Co}_{3}(4.7 \mathrm{gm}$. for each liter of $\mathrm{CO}_{2}$ desired) is placed in a glass in the bottom of the jar and $10 \%$ sulphuric acid is added. The jar is tightly sealed as soon as the reaction is complete. Cultures made in the morning will be found to have grown profusely by the next morning. It has been found possible to secure sufficient material from spinal fluid with 24 hours for agglutination reactions with various commercial serums to determine which serum is more suitable for the treatment of the case in question.

The following method of agglutinating has proved satisfactory. As many rows of 7 small test tubes are placed in the rack as there are strains to agglutinate. The first tube of each row is numbered with the number of

2 Arch. Int. Med., 1918, 21, p. 541.

s Jour. Am. Med. Assn., 1918, 70, p. 684. 
the corresponding slant. To each of the numbered tubes $2 \mathrm{cc}$ of salt solution is added; $0.8 \mathrm{cc}$ to the last tube, the salt solution control. In the second row place $0.8 \mathrm{cc}$ of a $1: 80$ dilution of diagnostic polyvalent meningococcic serum. In the third row place $0.8 \mathrm{c} c$ of a 1:80 dilution of a diagnostic normal meningococcic serum. In the fourth row place $0.8 \mathrm{cc}$ of a $1: 80$ dilution of an intermediate diagnostic serum. In the fifth row place $0.8 \mathrm{cc}$ of a 1:80 dilution of a diagnostic parameningococcic serum. In the sixth row

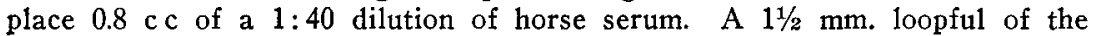
suspected organism is then scraped off a slant and emulsified in the tube containing $2 \mathrm{cc}$ of salt solution. To each of these diluted serums are added $0.2 \mathrm{cc}$ of the bacterial emulsion, the tubes are shaken and placed in the $56 \mathrm{C}$. incubator; readings are made in 12-24 hours. By using this method it is possible to secure a type determination without subculturing. Original slants may be subcultured after agglutination has proven it to be a meningococcus.

Table 1.-Meningitis Cases, Convalescents, Contacts and Noncontacts

\begin{tabular}{c|c|c|c|c}
\hline $\begin{array}{c}\text { Number } \\
\text { Throat Cultures } \\
\text { Taken }\end{array}$ & \multicolumn{2}{|c|}{ Positive in Air } & \multicolumn{2}{|c}{ Positive in CO } \\
\cline { 2 - 5 } & Number & Percentage & Number & Percentage \\
\hline 17 & 3 & 17.7 & 10 & 59.0 \\
53 & 2 & 3.7 & 9 & 16.0 \\
32 & 0 & 0.0 & 4 & 12.4 \\
36 & 0 & 0.0 & 3 & 8.3 \\
70 & 1 & 1.4 & 9 & 12.7 \\
12 & 0 & 0.0 & 0 & 0.0 \\
35 & 0 & 0.0 & 4 & 31.3 \\
120 & 0 & 0.0 & 1 & 3.7 \\
37 & 0 & 1.1 & 3 & 3.4 \\
88 & 1 & 1.4 & 54 & 10.8 \\
\hline 500 & 7 & & & \\
\hline
\end{tabular}

The following method has been used for carrier work:

A straight wire possessing moderate elasticity, about 10 inches long with cotton applicator at tip is used in preference to the West tube. These wire swabs were made up in lots of 8 each, placed in test tubes and sterilized. When ready for use one is withdrawn, bent to an angle of $45^{\circ}$ about $1 \frac{1 / 2}{2}$ inches from the tip by using the mouth of the test tube. The wire is then bent once again to a right angle 3 inches from the opposite end of the cotton applicator. This affords a handle and allows the swab to be used with greater dexterity. Cultures are secured by passing the swab into the nasopharynx by way of the mouth. Inoculations are made on one-half of each of 2 blood-agar plates, and one plate is incubated in the usual manner; the other at partial oxygen tension. The medium used is composed of $5 \%$ defibrinated human blood, not laked, $1 \%$ dextrose in $2 \%$ agar, and reaction plus 0.3 . The plates are incubated at $37 \mathrm{C}$. over night and examined the next morning. This is an approximate incubation period of 18 hours. Plates which show suspicious colonies are separated for picking, the others discarded. Quite frequently the aerobic plates may fail to disclose colonies that resemble meningococcus colonies whereas in the duplicate plate grown in $\mathrm{CO}_{2}$ they appear quite numerous. In the large majority of plates it has been evident that the colonies grown in $\mathrm{CO}_{2}$ were more luxuriant and vigorous than the aerobic 
ones. Suspicious colonies are transferred to blood-agar slants and, after incubation, examined by gram stain and agglutination. Cultures originally grown in $\mathrm{CO}_{2}$ have been carried in that atmosphere throughout.

Table 1 contrasts the percentage of carriers detected by the new and by the old methods.

Cultures of the last 15 spinal fluids showing gram-negative diplococci microscopically gave positive cultures in 13 of the cases, all cultured in $\mathrm{CO}_{2}$.

SUMMARY

Attention is again called to the fact that the meningococcus is a microaerophil.

The optimum reduction in oxygen tension for meningococcus cultures has been measured.

Simple, rapid and accurate methods for the isolation and cultivation of the meningococcus have been described.

Meningococci grow best in an atmosphere composed of approximately $10 \% \mathrm{CO}_{2}$ and $90 \%$ air.

In 500 nasopharyngeal cultures $10.8 \%$ carriers have been found by $\mathrm{CO}_{2}$, and $1.4 \%$ by the usual method. 